Check for updates

1 University of Bath, UK

2 Defence Science and Technology Laboratory, Salisbury, UK

3 The BMJ, London, UK

4 School of Civil Engineering, University of Leeds, UK

5 Royal Academy of Engineering, London, UK

6 Health and Safety Executive, Buxton, UK

7 Centre for Climate Repair at Cambridge, University of Cambridge, UK

8 Winton Centre for Risk and Evidence Communication, University of Cambridge, UK

Correspondence to: H Rutter h.r.rutter@bath.ac.uk Cite this as: BMJ 2021;375:e065312 http://dx.doi.org/10.1136/bmi-2021-065312 Published: 1 December 2021

\section{Visualising SARS-CoV-2 transmission routes and mitigations}

\section{Harry Rutter and colleagues reflect on the challenges of conveying uncertain estimates for viral transmission in a complex system}

\author{
Harry Rutter, ${ }^{1}$ Simon Parker, ${ }^{2}$ Will Stahl-Timmins, ${ }^{3}$ Catherine Noakes, ${ }^{4}$ Alexandra Smyth, ${ }^{5}$ Ron Macbeth, \\ Shaun Fitzgerald, ${ }^{7}$ Alexandra L J Freeman ${ }^{8}$
}

Scientific understanding about the epidemiology, genetics, and continuing evolution of covid-19 has been transformed since the disease was first identified at the end of 2019, but evidence on how and where the virus transmits remains limited and evolving. As covid-19 moves from pandemic to endemic, and many countries start to lift restrictions, individuals and organisations are increasingly faced with difficult personal and policy choices, such as how to make a workplace or a public area as safe as it can be while still being open and functional, or how to protect yourself and others. Such decisions continue, of necessity, to be made under considerable uncertainty. Clear communication is needed to help decision makers navigate this uncertainty.

To make informed decisions, whether about covid or anything else, everyone-from members of the public to policy makers-needs access to easily understandable, relevant descriptions of the best evidence available. Many approaches have been developed to communicate quantified evidence to help decision making for health, including tabular formats such as fact boxes, ${ }^{1-5}$ graphical formats such as icon arrays, ${ }^{6-8}$ and contextual aids such as risk ladders. ${ }^{10}$

Health related evidence incorporates a degree of epistemic uncertainty stemming from gaps in knowledge. In some cases, the use of large, well designed randomised controlled trials may help quantify this uncertainty and it can be communicated as a range ${ }^{11}$ alongside indicators of the unquantified uncertainties, such as the quality of the underlying evidence. ${ }^{12-16}$ However, in many cases-particularly for complex public health problems-the complexity of relations between causal factors may mean that there is not only high epistemic uncertainty about the variables in the system, and about how they interact, but also a high degree of variability due to individual and environmental factors. This is a major challenge in describing the transmission and mitigation of SARS-CoV-2, for which the multiple variables cannot be precisely quantified as they are contingent both on one another and on the specific characteristics of the complex adaptive systems within which they are considered.

\section{Evidence on transmission routes}

Understanding the current evidence around SARS-CoV-2 transmission and mitigation is an essential step towards taking informed decisions on protective measures, but there have been few attempts to collate and communicate the multiple factors that determine transmission, or to illustrate them in a way that systematically represents how different variables are contingent on one another. Many studies have looked at the factors that affect one particular transmission route, such as the influence of ventilation on airborne transmission, ${ }^{17}$ and a small number have grouped factors such as ventilation, face coverings, and behaviours into simple risk frameworks. ${ }^{18}$ However, there is little that considers all of the transmission routes and environmental and behavioural mitigations together; this is unsurprising given the difficulties of representing such poorly quantified and interacting variables.

There are many reasons for the paucity of robust empirical evidence on transmission of SARS-CoV-2 and the effectiveness of mitigation measures. It is extremely difficult to measure infectious SARS-CoV-2 virus in real world contexts, especially when airborne. Even if the virus were easily detectable, it remains infectious outside the body for only a relatively short period, far shorter than the time taken for an outbreak to become apparent and the relevant environment examined. Transmission is contingent on multiple factors, including the viral load of the infected person; their symptoms; the characteristics of their respiratory and other behaviours; physical configuration and other aspects of the transmission location such as ventilation, temperature, and humidity; the specific nature of any mitigation measures such as quality of any face covering or the methods used to clean surfaces; behavioural responses and adaptations in light of mitigation measures; and wider contextual factors such as population prevalence, vaccination, and immunity levels of those exposed.

Despite the difficulties of precise quantification, much is known, qualitatively, about SARS-CoV-2 transmission. It is widely accepted to occur through three major routes, all arising from the respiratory tract of an infectious person. ${ }^{19}$ When that person breathes, speaks, coughs, talks, or sings, particles which can potentially carry the virus are emitted in a continuum of aerosol and droplet sizes, from very small ( $<10 \mu \mathrm{m}$ diameter), through medium (10-100 $\mu \mathrm{m})$, to large $(>100 \mu \mathrm{m}) .^{2021}$

Transmission at close proximity to an infectious person is likely to be through a combination of mechanisms of exposure through inhalation of all sizes of particles up to around $100 \mu \mathrm{m}$, together with possible exposure to larger ballistic droplets that could land directly on mucous membranes in the eyes, nose, or mouth. ${ }^{18} 19$ 
Long range airborne transmission can occur through exposure to the smallest particles. These remain suspended in the air, travelling beyond $2 \mathrm{~m}$ from the source through airflows within an indoor environment, particularly if the ventilation rate is low. ${ }^{17} 22$

Transmission through surfaces or fomites seems to be less common than through inhalation 2324 but could potentially occur when surfaces are contaminated by deposition of larger particles or directly from contact with an infected person's hands. ${ }^{25}$ Virus particles can then be passed by subsequent touch onto the hands of a susceptible person, and then into their eyes, mouth, or nose. ${ }^{26}$ Possible variations on these routes include direct contact from an infected person who has contaminated their fingers from their own nose or mouth. Although faecal, urinary, and sexual transmission are theoretically possible, ${ }^{27-31}$ none has been confirmed in humans.

Despite extensive knowledge about the physics of aerosols, the absence of unequivocal empirical evidence on the amount of virus carried by different particle sizes, the relative contributions of surface, droplet, and airborne spread, or on the effectiveness of different mitigation measures has led to considerable uncertainties and difficulties in achieving scientific consensus. This has impeded clear communication of the roles of the different pathways in different situations.

Initial guidance from the World Health Organization emphasised the importance of aerosol transmission only in the limited case of "aerosol generating procedures"-such as endotracheal intubation or bronchoscopy-in healthcare settings. ${ }^{32}$ The importance of airborne transmission more generally was highlighted in a letter signed by 239 international scientists. ${ }^{33}$ Subsequent WHO guidance has acknowledged the possibility of aerosol spread in confined indoor spaces. ${ }^{32} 34$ A systematic review commissioned by WHO (at the time of writing still in preprint and not approved by two of three reviewers) concluded: "The lack of recoverable viral culture samples of SARS-CoV-2 prevents firm conclusions to be [sic] drawn about airborne transmission." 35 The preprint prompted a comment article in the Lancet in April 2021 arguing that "there is consistent, strong evidence that SARS-CoV-2 spreads by airborne transmission."36

At the heart of the challenge of communicating the relative importance of these transmission routes and their potential mitigation methods to decision makers (including the public) has been the uncertainty around quantification of the absolute likelihoods of transmission, and the amount of variation in them because of different conditions. However, communication of evidence, and decision making based on it, does not inherently require precise quantification: it can be based on relatively "fuzzy" estimates, appropriately conveyed. Such quantification-which should include uncertainties based on both quantified and unquantifiable sources as well as individual variation-should take into account both qualitative and quantitative expert knowledge, as well as empirical evidence.

\section{Visualising transmission routes}

Even if "hard" empirical evidence is absent, it is still important for people to be able to make decisions based on the best available knowledge about a topic. To support such decision making, we conducted an exercise to elicit expert opinions on the state of knowledge about SARS-CoV-2 transmission (box 1), and then to represent these opinions in as intuitive and useful a way as possible using an interactive tool (box 2). Our aim was for the tool to summarise and communicate the best available evidence-including the expert knowledge that lies outside of quantified uncertainty ranges-while also conveying the evidential uncertainty and variability, and the disagreement that exists between experts about some pathways and interventions.

Box 1: Eliciting uncertain evidence of viral transmission and mitigation from experts

Having devised a simple illustration of the most likely transmission pathways for SARS-CoV-2, we wanted to be able to give the public and decision makers an indication of expert opinion on the relative importance of each, and the effects of different mitigations.

We used a conceptual model of transmission that includes seven respiratory activities, in two room sizes, each with or without ventilation, as well as outdoor environments, and three forms of contact on three types of surface, across six transmission pathways, with 10 possible mitigation interventions. The tool illustrates the relative importance of different transmission pathways, and the likely effects of mitigation measures on those pathways, in the context of different scenarios. These scenarios include the nature of respiratory activity concerned, ranging from the infected person being silent to coughing or singing. The tool thus demanded over 100 variables to be estimated, each of which needed to take into account not just uncertainty, but variability.

To obtain estimates that encompassed as much expert knowledge as possible, we used a two stage elicitation process during February and March 2021. This allowed us to bring together the current

knowledge-qualitative and quantitative-among international experts from a range of scientific disciplines on the likely rates of transmission along each pathway, a process we describe elsewhere. ${ }^{37}$ We chose this method specifically to provide the data needed for the interactive, online visualisation tool that we thought was missing in the guides available for decision makers. $38-40$

Our tool is designed to help individuals or risk managers considering a single encounter in which a susceptible person might come into contact with an infected person. It does not attempt to address population level factors that affect transmission, such as prevalence of infection, socioeconomic factors, or the level of vaccination; nor does it engage with individual level contextual factors such as vaccination status, occupation, or household composition. Although these factors are extremely important, they would have added considerable additional complexity and uncertainty. The outputs of the tool therefore need to be considered in the context of these wider factors.

We developed this tool before the dominance of the more transmissible delta variant, and new evidence relating to transmission is published all the time. If we were to repeat the exercise in the context of more recent variants of concern, some of the relative roles of different transmission routes and mitigation measures might have increased or decreased in importance, but the core features of the visualisation are unlikely to have changed substantially. If important evidence were to arise that invalidated any elements of the tool it could be amended.

\section{Box 2: Representing scenarios visually}

One core task was to devise a method for clearly communicating uncertainties, imprecise quantities, and expert disagreements in a way that still proved useful for decision makers. In addition to uncertainty about potential transmission routes and mitigations, there is a great degree of variability and contingency within every scenario represented. Individuals will differ in their viral loads, their breathing and speech patterns, and their behaviour; environments will differ in factors such as ventilation rates, airflow patterns, temperatures, and humidity. All of these could affect the likelihood of transmission, which is itself a probabilistic event. These uncertainties all needed to be considered in the tool.

The interactive graphic we developed (see online at www.bmj.com/content $/ 375 / \mathrm{bmj}-2021-065312$ ) shows the majority opinion of experts in the final round of the elicitation process for most variables. It was deliberately not intended to represent a precise quantitative model for viral transmission. Instead, it is designed primarily as a communication tool to aid decision making: providing a simplified model of the transfer of virus between two individuals in order to allow the user easily to compare the likely relative importance of transmission routes in different 
environments, with or without various mitigation measures. The gender of the people depicted is allocated randomly by the tool.

To prevent unwarranted assumptions about precision based on the diagram, and to convey an appropriately high level of uncertainty, we used a smooth colour gradient to represent the expert elicited values. Colour is both intuitive for audiences to interpret and, with a smooth gradient, can communicate imprecise values while allowing general comparisons of relative values..$^{41}$ Thus, to illustrate relatively higher transmission rates we show darker coloured routes, and vice versa, but these tones should not be seen as representing precisely quantified levels.

For those interested in the actual estimated uncertainty ranges (including epistemic and aleatory uncertainties, as well as expected variation within a scenario), each "node" in the diagram, each mitigation, and each scenario, was given a small blue "i" button. When selected, these display the size of the quantified uncertainty range elicited from experts for each variable, as well as their estimates of the quality of the evidence base for each variable (on a 1-5 scale), and a text description of the causes of uncertainty and variability that were listed. Where there were differences of opinion among groups of experts, their alternative opinions are also shown in the form of ranges in the same pop-up window.

Given the large number of parameters, with appreciable uncertainty and variability around all of them, and the extreme difficulty of measuring almost any of them in a precise or consistent way, even a richly populated empirical evidence base would struggle to allow a systematic review that could generate meaningful findings to underpin the visualisation tool. The severely constrained empirical evidence base that we would have had to rely on could not have provided the information necessary for the tool. A formal expert elicitation exercise provided an appropriate way to obtain the required information given not only the infeasibility of basing the tool on a systematic review of empirical research, but also the amount of knowledge held within the expert community that is not reflected in published quantitative evidence.

Expert elicitation allowed us both to adduce estimates of transmission risks across the multiple different scenarios under consideration and to identify the levels of agreement and uncertainty of each estimate, both of which are displayed within the tool. While the empirical evidence base is not directly represented within the tool, it is embodied within the responses of the experts who contributed their knowledge, including providing references to evidence that they knew about.

Notwithstanding disagreements revealed by the expert elicitation exercise, the inhalation routes dominate in almost all situations, and face coverings, especially when worn by an infected person as a form of source control, are the most important mitigation measure. However, it is important to note that all routes were considered to play a part in transmission, and simple measures such as physical distancing, hand washing, and respiratory hygiene make a useful contribution: the fact that specific transmission routes and mitigations are relatively more important in some situations does not remove the need to consider all relevant transmission routes and mitigations in all situations.

One source of uncertainty that we had not anticipated sufficiently was disagreement between experts. Opinions concerning the role of aerosol transmission varied widely in the elicitation exercise, but we also found divergent views on several other variables, such as the amount of small particle inhalation at different room sizes; the effects of different kinds of masks on inhaled aerosols; and the effects of face coverings on transfer from hand to eyes, nose, and mouth. Some of this variation may be the result of different epistemological perspectives, with some people prioritising empirical data specific to SARS-CoV-2, while others placed a greater emphasis on robust theory and generalisable evidence from other contexts. We incorporated this expert disagreement information into the graphic in a deeper layer, where viewers can click to see alternative views. Here they can also see the embedded uncertainties-how highly experts rated the quality of evidence on that particular variable.

\section{Reducing quantifiable uncertainty}

The continued diversity of expert views more than 18 months since covid-19 was first described reflects both the complexity of the methodological challenges and a lack of sufficient interdisciplinary and strategic research during and before the pandemic. Generating robust evidence on the complex and highly contingent routes of transmission of SARS-CoV-2 is not straightforward, but the impressive scientific attention paid to vaccines and viral genomics must be matched by an equivalent focus on research to increase our understanding of the ways in which SARS-CoV-2 behaves, how it is transmitted, and how we can most effectively reduce the likelihood of transmission through individual, local, and societal level interventions.

Improving our response to the next pandemic will require a suite of actions, with many of them relating to the capacity, structures, and resources devoted to research. But equally important will be a willingness to embrace a range of scientific perspectives, with a focus on identifying the most relevant and appropriate evidence available to guide decision making, whether it is based on empirical research or theory, and finding the balance between qualitative and quantitative data. The same is true of many complex public health challenges, in which it is rarely possible to rely on the kinds of high quality, high precision quantification methods that are used to evaluate pharmaceuticals or surgical procedures. ${ }^{42}$

We hope that this visualisation tool will be useful for exploring transmission routes in a transparent and interactive way, and will help guide people making decisions about which mitigation measures might be most effective to protect themselves and others from the continued challenges that this virus presents. We also hope that the approach we have taken both to eliciting and communicating knowledge will prove helpful to those faced with the challenge of communicating complex, imprecise, and uncertain evidence in the future.

\section{Key messages}

- There is a paucity of robust quantitative evidence on the importance of different mechanisms of transmission of SARS-CoV-2, or on the effectiveness of environmental and behavioural mitigation measures in a broad range of real-world environments.

- Communicating evidence that has broad uncertainties or is difficult to quantify, such as transmission of SARS-CoV-2, presents particular challenges

- In the absence of robust quantitative evidence, expert elicitation exercises can help to collate and synthesise knowledge from multiple sources

- Expert elicitation was used to underpin an interactive tool to visualise the ways in which SARS-CoV-2 is transmitted and the likely effects of mitigation measures in different contexts

- The visualisation tool helps to convey inherent uncertainties in the data, while providing a means to explore the relative influence of different mitigation measures

- This tool should support decision makers and the public to make informed decisions about how best to reduce transmission of SARS-CoV-2 in different contexts 
Contributors and sources: The original transmission model was developed by HR, CN, SP, AS, ALJF, RM, and SF. HR, ALJF, SP and CN then worked with WST to develop the interactive graphic, which was shared with the other authors. HR, CN, SP, AS, ALJF, RM, SF are all members of the environment and modelling subgroup of the UK Government Strategic Advisory Group for Emergencies (SAGE EMG), from which they formed a working group to develop this project. WST is data graphics designer. HR wrote the first version of the manuscript, and all other authors contributed to refining it. HR is the guarantor.

Competing interests: We have read and understood BMJ policy on declaration of interests and declare the following: SF is a co-investigator on the UKRI AIRBODS research programme, CN is principle investigator on the UKRI TRACK, Breathing Cities and HECOIRA projects and co-investigator on the NIHR Contact study. SP is co-investigator on the UKRI TRACK project. HR, SF, AS, CN were on the working group for the Royal Academy of Engineering Infection Resilient Environments report. CN was on the working group for the Academy of Medical Sciences reports on covid in 2020 and 2021. WST is employed by The BMJ but was not involved in, or privy to, any aspects of editorial decision making for this article or the accompanying paper in BMJ Open.

Provenance and peer review: Not commissioned; externally peer reviewed.

We thank the 26 experts who provided their views as part of the elicitation study. ${ }^{37}$ Funding to support development of the visualisation tool and publication was provided the Protect Covid-19 National Core Study on transmission and environment, managed by the Health and Safety Executive on behalf of HM Government. The tool was user tested by the Winton Centre for Risk and Evidence Communication, funded by a donation from the David and Claudia Harding Foundation.

1 Woloshin S, Schwartz LM. Communicating data about the benefits and harms of treatment: a randomized trial. Ann Intern Med 2011;155:87-96.

doi: 10.7326/0003-4819-155-2-201107190-00004 pmid: 21768582

2 Schwartz LM, Woloshin S. The drug facts box: improving the communication of prescription drug information. Proc Natl Acad Sci U S A 2013;110(Suppl 3):14069-74. doi: 10.1073/pnas.1214646110 pmid: 23942130

3 McDowell M, Rebitschek FG, Gigerenzer G, Wegwarth O. A simple tool for communicating the benefits and harms of health interventions: a guide for creating a fact box. MDM Policy Pract 2016;1:2381468316665365. doi: 10.1177/2381468316665365 pmid: 30288405

4 McDowell M, Gigerenzer G, Wegwarth O, Rebitschek FG. Effect of tabular and icon fact box formats on comprehension of benefits and harms of prostate cancer screening: a randomized trial. Med Decis Making 2019;39:41-56. doi: 10.1177/0272989X18818166 pmid: 30799691

5 Brick C, McDowell M, Freeman ALJ. Risk communication in tables versus text: a registered report randomized trial on "fact boxes." $R$ Soc Open Sci2020;7:190876.

doi: 10.1098/rsos.190876 pmid: 32269779

6 Lipkus IM. Numeric, verbal, and visual formats of conveying health risks: suggested best practices and future recommendations. Med Decis Making 2007;27:696-713. doi: 10.1177/0272989X07307271 pmid: 17873259

7 Zipkin DA, Umscheid CA, Keating NL, etal. Evidence-based risk communication: a systematic review. Ann Intern Med 2014;161:270-80. doi: 10.7326/M14-0295 pmid: 25133362

8 Galesic M, Garcia-Retamero R, Gigerenzer G. Using icon arrays to communicate medical risks: overcoming low numeracy. Health Psychol 2009;28:210-6. doi: 10.1037/a0014474 pmid: 19290713

9 Paling J. Strategies to help patients understand risks. BMJ 2003;327:745-8. doi: 10.1136/bmj.327.7417.745 pmid: 14512489

10 Freeman ALJ, Kerr J, Recchia G, etal. Communicating personalized risks from COVID-19: guidelines from an empirical study. R Soc Open Sci2021;8:201721. doi: 10.1098/rsos.201721 pmid: 33996117

11 van der Bles AM, van der Linden S, Freeman ALJ, Spiegelhalter DJ. The effects of communicating uncertainty on public trust in facts and numbers. Proc Natl Acad Sci U S A 2020;117:7672-83. doi: 10.1073/pnas.1913678117 pmid: 32205438

12 Guyatt GH, Oxman AD, Kunz R, Vist GE, Falck-Ytter Y, Schünemann HJGRADE Working Group. What is "quality of evidence" and why is it important to clinicians?BMJ 2008;336:995-8. doi: 10.1136/bmj.39490.551019.BE pmid: 18456631

13 Atkins D, Eccles M, Flottorp S, etalGRADE Working Group. Systems for grading the quality of evidence and the strength of recommendations I: critical appraisal of existing approaches: the GRADE working group. BMC Health Serv Res 2004;4:38. doi: 10.1186/1472-6963-4-38 pmid: 15615589

14 Guyatt GH, Oxman AD, Kunz R, Vist GE, Falck-Ytter Y, Schünemann HJ. What is 'quality of evidence' and why is it important to clinicians?BMJ2008;336:995-8. doi: 10.1136/bmj.39490.551019.BE pmid: 18456631

15 Hultcrantz M, Rind D, AkI EA, etal. The GRADE working group clarifies the construct of certainty of evidence. J Clin Epidemio/2017;87:4-13. doi: 10.1016/j.jclinepi.2017.05.006 pmid: 28529184

16 Akl EA, Maroun N, Guyatt G, etal. Symbols were superior to numbers for presenting strength of recommendations to health care consumers: a randomized trial. J Clin Epidemiol 2007;60:1298-305. doi: 10.1016/j.jclinepi.2007.03.011 pmid: 17998085

17 Miller SL, Nazaroff WW, Jimenez IL, etal. Transmission of SARS-CoV-2 by inhalation of respiratory aerosol in the Skagit Valley Chorale superspreading event. Indoor Air 2021;31:314-23. doi: 10.1111/ina.12751 pmid: 32979298

18 Jones NR, Qureshi ZU, Temple RJ, Larwood JPJ, Greenhalgh T, Bourouiba L. Two metres or one: what is the evidence for physical distancing in covid-19?BMJ2020;370:m3223.

doi: 10.1136/bmj.m3223 pmid: 32843355
19 Milton DK. A rosetta stone for understanding infectious drops and aerosols. J Pediatric Infect Dis Soc 2020;9:413-5. doi: 10.1093/jpids/piaa079 pmid: 32706376

20 Duguid JP. The size and the duration of air-carriage of respiratory droplets and droplet-nuclei. J Hyg (Lond) 1946:44:471-9. doi: 10.1017/S0022172400019288 pmid: 20475760

21 Johnson GR, etal. Modality of human expired aerosol size distributions. J Aerosol Sci 2011;:42:839-51doi: 10.1016/j.jaerosci.2011.07.009.

22 Jones B, Sharpe P, Iddon C, Hathway EA, Noakes CJ, Fitzgerald S. Modelling uncertainty in the relative risk of exposure to the SARS-CoV-2 virus by airborne aerosol transmission in well mixed indoor air. Build Environ2021;191:107617. doi: 10.1016/j.buildenv.2021.107617 pmid: 33495667

23 Onakpoya IJ, Heneghan C), Spencer EA, et al. 2021 SARS-CoV-2 and the role of fomite transmission: a systematic review. [Preprint.] F1000 Res 2021. doi: 10.12688/f1000research.51590.2

24 Port JR, Yinda CK, Owusu IO, etal. SARS-CoV-2 disease severity and transmission efficiency is increased for airborne compared to fomite exposure in Syrian hamsters. Nat Commun 2021;12:4985. doi: 10.1038/s41467-021-25156-8 pmid: 34404778

25 Kraay ANM, Hayashi MAL, Hernandez-Ceron N, etal. Fomite-mediated transmission as a sufficient pathway: a comparative analysis across three viral pathogens. BMC Infect Dis 2018;18:540. doi: 10.1186/s12879-018-3425-x pmid: 30373527

26 King M-F, Wilson AM, Weir MH, etal. Modeling fomite-mediated SARS-CoV-2 exposure through personal protective equipment doffing in a hospital environment. Indoor Air 2021. doi: 10.1111/ina.12938 pmid: 34693567

27 Dergham J, Delerce J, Bedotto M, La Scola B, Moal V. 2021 Isolation of viable SARS-CoV-2 virus from feces of an immunocompromised patient suggesting a possible fecal mode of transmission. J Clin Med 2021;10:2696. doi: 10.3390/jcm10122696 pmid: 34207314

28 Meng XJ, Liang TJ. SARS-CoV-2 infection in the gastrointestinal tract: fecal-oral route of transmission for covid-19? Gastroenterology 2021;160:1467-9. doi: 10.1053/j.gastro.2021.01.005 pmid: 33422479

29 Jiao L, Li H, Xu J, etal. The gastrointestinal tract is an alternative route for SARS-CoV-2 infection in a nonhuman primate model. Gastroenterology 2021;160:1647-61. doi: 10.1053/j.gastro.2020.12.001 pmid: 33307034

30 Jeong HW, Kim SM, Kim HS, etal. Viable SARS-CoV-2 in various specimens from COVID-19 patients. Clin Microbiol Infect 2020;26:1520-4. doi: 10.1016/j.cmi.2020.07.020 pmid: 32711057

31 Morelli F, Meirelles LEF, de Souza MVF, etal. Covid-19 infection in the human reproductive tract of men and nonpregnant women. Am J Trop Med Hyg 2021;104:814-25. doi: 10.4269/aitmh.20-1098 pmid: 33534765

32 World Health Organization. 2020 Modes of transmission of virus causing covid-19: implications for IPC precaution recommendations. 27 Mar 2020 (updated 9 Jul 2020).

https://www.who.int/news-room/commentaries/detail/modes-of-transmission-of-virus-causingcovid-19-implications-for-ipc-precaution-recommendations

33 Morawska L, Milton DK. It is time to address airborne transmission of coronavirus disease 2019 (covid-19). Clin Infect Dis 2020;71:2311-3. doi: 10.1093/cid/ciaa939 pmid: 32628269

34 World Health Organization. Coronavirus disease (COVID-19): How is it transmitted? 2021. https://www.who.int/news-room/q-a-detail/coronavirus-disease-covid-19-how-is-it-transmitted

35 Heneghan CJ, Spencer EA, Brassey J, et al. 2021 SARS-CoV-2 and the role of airborne transmission: a systematic review. [Preprint.] F1000 Resdoi: 10.12688/f1000research.52091.2

36 Greenhalgh T, Jimenez JL, Prather KA, Tufekci Z, Fisman D, Schooley R. Ten scientific reasons in support of airborne transmission of SARS-CoV-2. Lancet 2021;397:1603-5. doi: 10.1016/S0140-6736(21)00869-2 pmid: 33865497

37 Freeman ALJ, Parker S, Noakes C, etal. Expert elicitation on the relative importance of possible SARS-CoV-2 transmission routes and the effectiveness of mitigations. BMJ Open 2021;11:0050869. doi: 10.1136/bmjopen-2021-050869 pmid: 34853105

38 West R, Michie S, Rubin G), Amlôt R. Applying principles of behaviour change to reduce SARS-CoV-2 transmission. Nat Hum Behav 2020;4:451-9. . doi: 10.1038/s41562-020-0887-9 pmid: 32377018

39 Salas J, Almodóvar L. 2021 A room, a bar and a classroom: how the coronavirus is spread through the air. El Pais. See https://english.elpais.com/society/2020-10-28/a-room-a-bar-and-a-classhow-the-coronavirus-is-spread-through-the-air.html.

40 Umakanthan S, Sahu P, Ranade AV, etal. Origin, transmission, diagnosis and management of coronavirus disease 2019 (COVID-19). Postgrad Med J 2020;96:753-8.

doi: 10.1136/postgradmedj-2020-138234. pmid: 32563999

41 Spiegelhalter D, Pearson M, Short I. Visualizing uncertainty about the future. Science 2011;333:1393-400. doi: 10.1126/science.1191181 pmid: 21903802

42 Rutter H, Savona N, Glonti K, etal. The need for a complex systems model of evidence for public health. Lancet 2017;390:2602-4. doi: 10.1016/S0140-6736(17)31267-9 pmid: 28622953 